\title{
Pegylated Recombinant Interleukin-2 THOR-707
}

National Cancer Institute

\section{Source}

National Cancer Institute. Pegylated Recombinant Interleukin-2 THOR-707. NCI

Thesaurus. Code C162645.

A pegylated recombinant, eng ineered variant of cytokine interleukin-2 (IL-2; IL2) where novel amino acid is encoded in the IL-2 gene that is leveraged for use in site-specific pegylation, with potential immunostimulating activity. Upon administration of pegylated recombinant IL-2 THOR-707, the IL-2 variant moiety binds to dimers containing the IL-2 receptor beta and gamma chains (IL2Rbg; IL2Rbetagamma) on immune cells, such as cytotoxic T-lymphocytes (CTLs) and natural killer (NK) cells, thereby activating these cells and inducing their expansion. It also induces the expression of certain cytotoxic cytokines, such as interferon-gamma (IFNg) and transforming growth factor-beta (T GFb). The specific induction of T-cell-mediated cytotoxic immune responses against tumor cells causes tumor cell destruction. The addition of the novel amino acid and the concurrent pegylation prevents the binding of the IL-2 moiety to the IL-2 receptor alpha chain (IL2Ra), thereby blocking the IL2Ra-mediated activation of CD4-positive regulatory and immunosuppressive T-cells (Tregs). Since it cannot bind to IL2Ra expressed on innate lymphoid cells in the vascular endothelium, THOR-707 prevents IL-2-mediated recruitment and activation of eosinophils, and inhibits the induction of eosinophilmediated vascular leak syndrome (VLS). Pegylation also extends the half-life of THOR707. Compared to recombinant IL-2, THOR-707 allows for increased IL-2Rbg binding and proliferation of CT Ls without stimulating the toxicity caused by binding to IL-2Ra. 